\title{
Felicidade, bem-estar digital e segurança digital infantil - comunicação do Ministério da Felicidade do Dubai e dos Emirados Árabes Unidos em 2019.
}

Happiness, digital wellbeing and child digital safety - Dubai and United Arab Emirates Ministry of Happiness Communication in 2019

Diamantino Ribeiro ${ }^{1}$, António Pedro Costa² e Jorge Remondes ${ }^{3}$

${ }^{1}$ CEFAGE, Universidade de Évora, Portugal, Email: diamantinojtribeiro@gmail.com, ORCID: https://orcid.org/0000-0002-7168-8821

2 Departamento de Educação e Psicologia, da Universidade de Aveiro, Portugal, Email: apcosta@ua.pt, ORCID: https://orcid.org/0000-0002-4644-5879

${ }^{3}$ CETRAD/UTAD - Centro de Estudos Transdisciplinares para o Desenvolvimento da Universidade de Trás-os-Montes e Alto Douro, Portugal, Email: jorge@jorgeremondes.eu, ORCID: https://orcid.org/0000-0002-5259-4379

\author{
ART I C LE IN F O \\ Article history: \\ Received 2021-02-22 \\ Accepted 2021-05-04 \\ Available online 2021-05-04
}

Palavras-chave: Felicidade. Segurança Digital. Dubai. Bem-Estar. Emirados Árabes Unidos.

Keywords: Happiness. Digital Safety. Dubai. Wellness. UAE.

RESUMO. Os conceitos de felicidade, positividade e bem-estar no Dubai e nos Emirados Árabes Unidos fazem parte das políticas governamentais. O Dubai tem trabalhado para implementar a sua visão e objetivo de ser a cidade mais feliz do planeta. Aparentemente trata-se apenas de emoções, mas envolve muita tecnologia e iniciativas inovadoras. Para atingir esse objetivo, existe um Ministério da Felicidade, uma Agenda da Felicidade, um Programa Nacional de Felicidade e Positividade, um website 'Happy Dubai', uma plataforma 'Smart Dubai', etc. Temos estudado a felicidade como política no Dubai desde o lançamento de uma aplicação para medir os níveis de felicidade dos cidadãos em 2014, bem como a comunicação governamental do Ministério da Felicidade desde 2016. Este artigo aborda os programas e iniciativas do Ministério da Felicidade em 2019. Ao longo do estudo da comunicação relativa ao ano de 2019, a nossa atenção foi direcionada para o tema da Segurança Digital Infantil - um tema emergente e urgente nas agendas das políticas digitais em todo o mundo. Este artigo é uma síntese do estudo exploratório sobre Felicidade como política governamental, sobre o Bem-estar Digital e sobre a Iniciativa de Segurança Digital Infantil dos Emirados Árabes Unidos. Entre os aspetos mais relevantes do estudo realizado, destacamos o facto de a Ministra da Felicidade continuar a trabalhar ativamente para construir o caminho para a cidade mais feliz do planeta. Relativamente à Child Digital Safety Initiative, uma ação integrada na Digital Wellness Strategy 2031, o principal objetivo é dotar as crianças com competências necessárias e ajudar os pais a compreender as ameaças do mundo digital, proporcionando-lhes os meios para as combater e para ajuda-los a gerir melhor a vida digital dos seus filhos. 
ABSTRACT. Happiness, positivity and wellbeing concepts in Dubai and the United Arab Emirates are considered government policies. Dubai's has been working to implement its vision and goal to be the happiest city on Earth. It's all about emotions but it involves a lot of technology and innovative initiatives. To achieve that goal, there is a Ministry of Happiness, a Happiness Agenda, a National Happiness and Positivity Program, a Happy Dubai webs page, a Smart Dubai platform, etc. We have been studying Happiness as a policy in Dubai since the launch of the happiness meter in 2014, and government communication of the Ministry of Happiness since 2016. This paper focuses on the programs and initiatives of the Ministry of Happiness in 2019. Along the study our attention was directed to the Child Digital Safety - an emergent and urgent topic on the digital policy agendas worldwide. This paper is an exploratory study about Happiness as a policy, Digital Wellness and UAE's Child Digital Safety Initiative. Among our key findings, we emphasize the fact that the Minister of Happiness works actively to build the path to the happiest city on Earth. Regarding the Child Digital Safety Initiative, an initiative integrated on the Digital Wellness Strategy 2031, the main goal is to provide children with the necessary skills, and assist parents in understanding the threats of the digital world, along with the means to counter them in order to better manage the digital life of their children.

\section{Introdução}

A história dos Emirados Árabes Unidos (EAU) é um caso de excecional sucesso. Desde a sua fundação em 1971, a Federação dos Emirados Árabes Unidos tem sindo pautada por marcos importantes em várias áreas, nomeadamente na educação, tecnologia e sociedade. A criação da Federação permitiu que o governo impulsionasse e implementasse na região verdadeiras revoluções económicas e urbanas, transformando os EAU num dos principais destinos turísticos e num polo de desenvolvimento e investimento da região (UAEGOV, 2009).

De acordo com a Comissão Nacional dos Objetivos de Desenvolvimento Sustentável (ODS) dos Emirados Árabes Unidos, no que diz respeito ao desempenho do setor público, os EAU têm um dos melhores desempenhos do mundo. O governo dos Emirados Árabes Unidos está empenhado em alcançar resultados excecionais em termos de economia, sociedade e meio ambiente. O governo tem uma visão muito definida para o desenvolvimento da Federação. Para cumprir a sua estruturada agenda, o governo confia nos seus competentes e eficientes organismos, orientados para trabalhar em conformidade com os mais altos padrões, baseados em modelos de desempenho e inovação (UAE NATIONAL COMMITTEE ON SDGS 2030, 2020).

Reconhecido por desenvolver e implementar estratégias e políticas inovadoras, em 2016 o Sheik Mohammed Al Maktoum anunciou a criação do Ministério da Felicidade e nomeou a Sra. Ohood Al Roumi como Ministra de Estado da Felicidade. Poucos dias depois, o Programa Nacional de Felicidade e Positividade foi aprovado pelo governo.

Em 2019, os Emirados Árabes Unidos criaram e aprovaram a Estratégia Nacional para o Bem-estar 2031; o novo programa tem um foco especial no bem-estar das gerações futuras (CABINET, 2019).

Depois de recolhermos e observarmos o conteúdo das notícias publicadas em 2019 sobre o Ministério da Felicidade do Dubai e dos Emirados Árabes Unidos, decidimos realizar um estudo preliminar sobre o Programa de Segurança Digital Infantil dos Emirados Árabes Unidos. O nosso objetivo principal é entender como o programa relaciona conceitos abstratos como a felicidade e o bem-estar com 
conceitos tecnológicos como bem-estar digital e de que forma todos os elementos se integram na Estratégia Nacional de Bem-estar 2031. De acordo com o governo e com as notícias publicadas, as iniciativas para promover o bem-estar digital e a segurança digital infantil foram planeadas e encontram-se em implementação (CABINET, 2019).

Temos vindo a estudar a comunicação governamental e, em particular do Ministério da Felicidade dos Emirados Árabes e do Dubai, a partir da análise das notícias publicadas em língua inglesa no corpus latente desde sua criação em 2016. Este trabalho tem uma base anual.

Os dados foram extraídos no corpus latente. Realizou-se uma recolha das notícias publicadas nos media online (por agências de notícias, jornais e pelo próprio governo) relativas ao Ministério da Felicidade e a iniciativas relacionadas, em lingua inglesa, durante o ano de 2019. Utilizamos a metodologia qualitativa, recorrendo às técnicas de observação e análise de conteúdo (BAZELEY, 2013; SCHREIER, 2008) para desenvolver o nosso estudo.

Em termos globais, podemos considerar que este estudo vem reforçar a visão de constante inovação do governo dos EAU, neste caso, através da iniciativa pioneira de introdução de políticas e estratégias, destinadas a promover e educar as gerações de nativos digitais e das suas famílias.

\section{A Felicidade como política governamental e o Programa Nacional de Felicidade e Positividade dos Emirados Árabes Unidos}

Os designados países em vias de desenvolvimento têm como modelo as nações que prosperam economicamente, nas quais o PIB (Produto Interno Bruto) é o principal objetivo da gestão governamental. No entanto, cada vez mais economias consideradas prósperas e países em desenvolvimento estão interessados em modelos e estratégias que promovam o bem-estar, a felicidade e positividade dos cidadãos (OISHI \& DIENER, 2014).

O Produto Interno Bruto (PIB) costumava ser o principal objetivo das políticas governamentais, em parte porque era um dos poucos índices que revelava claramente o desempenho do país. Mais recentemente, existem importantes pesquisas que incluem questões de felicidade, bem-estar ou qualidade de vida, com o objetivo de estudar a informação sobre uma sociedade feliz (ABDELATY et al., 2018; FREY; STUTZER, 2014; OISHI \& DIENER, 2014; RIBEIRO; LARANJEIRA, 2018; STIGLITZ, [s.d.]).

Um exemplo é o Relatório Mundial da Felicidade (World Happiness Report), que desde 2012, se tornou uma ferramenta importante para governos, organizações e académicos. O World Happiness Report é uma referência no que diz respeito aos referidos índices. Esta publicação, reúne informações de 156 países sobre as perceções dos cidadãos relativamente à felicidade, qualidade de vida e bem-estar (HELLIWELL, JOHN F., RICHARD LAYARD, JEFFREY SACHS, AND JANEMMANUEL DE NEVE, 2020); trata-se de um trabalho e, respetiva publicação, desenvolvido e coordenado por especialistas reconhecidos mundialmente.

A felicidade como política governamental, foi preconizada no Butão na década de 1970. A governação no Butão integra princípios singulares, como a espiritualidade e a compaixão é centenária. Esses princípios são colocados em prática pelos cidadãos e pelas organizações governamentais. O conceito de Felicidade Interna Bruta (FIB) foi desenvolvido pelo Rei Jigme Singye Wangchuck. Com o tempo, a visão foi sendo compartilhada com outros países, tornou-se mais estruturada e baseada em tecnologia. O Índice FIB, de acordo com os líderes e visão do Butão, traduz uma melhor noção da perceção dos cidadãos sobre a vida real (BHUTAN CENTRE, 2016). 
Inspirado pela visão do Butão, em 2014 o governo de Dubai foi pioneiro na introdução da Felicidade nas políticas governamentais. Na altura, o governo criou um "scorecard" para medir a Felicidade (um instrumento digital para analisar o nível de satisfação dos cidadãos e residentes com os serviços públicos). Alguns anos depois, - Sheik Mohammed Al Maktoum surpreendeu a região e o mundo ao anunciar a criação do Ministério da Felicidade do Dubai e dos Emirados Árabes Unidos. Desde então, o governo continua a inovar neste campo. Um Programa Nacional de Felicidade e Positividade foi implementado, foi criada uma web page designada 'happyuae.com', entre outras, tendo o governo posteriormente procedido à implementação de centenas de iniciativas relacionadas com a felicidade e positividade, quer nos órgãos do governo e instituições públicas, como em organizações privadas (Costa \& Ribeiro, 2019; C. \& R. Ribeiro 2020; D. Ribeiro, Costa, et al., 2020; D. Ribeiro, Remondes, et al., 2020).

De acordo com o Relatório Mundial da Felicidade de 2016, os Emirados Árabes Unidos (UAE) integraram a felicidade e o bem-estar como políticas de governo e têm vindo a desenvolver ações para cumprir o seu compromisso; o governo dos Emirados Árabes Unidos também criou oportunidades para promover a felicidade e o bem-estar enquanto política governamental em todo o mundo (HELLIWELL, JOHN F., RICHARD LAYARD, JEFFREY SACHS, AND JAN-EMMANUEL DE NEVE, 2020). Consequentemente, os Emirados Árabes Unidos têm também vindo a subir no ranking do Índice Mundial de Felicidade, especialmente após o compromisso de 2016 de implementar políticas de felicidade e bem-estar.

O compromisso do governo vai muito para além do tempo presente (AL MAKTHOUM, 2018; RIBEIRO, D., COSTA, A. P., \& REMONDES, 2020; RIBEIRO, 2020). Em 2016, de acordo com Abdulla Nasser Lootah (2019), os Emirados Árabes Unidos comprometeram-se com os seus cidadãos a trabalhar de forma que até 2071 a sociedade local viva no melhor país do mundo, de acordo com parâmetros e padrões internacionalmente reconhecidos (LOOTAH, 2019; UAE NATIONAL COMMITTEE ON SDGS 2030, 2020; UAEGOV, 2020).

Além disso, o objetivo dos Emirados Árabes Unidos é desempenhar um papel importante em termos mundiais, liderando e encorajando todos os países a trabalhar na "felicidade, coexistência pacífica, justiça social e dignidade para todos" (AL MAKTHOUM, 2018; UAE NATIONAL COMMITTEE ON SDGS 2030, 2020; UAEGOV, 2020).

Portanto, o governo está a tentar construir uma sociedade que tenha a felicidade, a prosperidade e o bem-estar dos indivíduos e da sociedade, como políticas centrais (UAE NATIONAL COMMITTEE ON SDGS 2030, 2020).

\section{As notícias sobre o Ministério da Felicidade em 2019 e a Estratégia Nacional de Bem-estar 2031.}

Aquando da constituição do Ministério da Felicidade e da nomeação Sra. Ohood bint Khalfan Al Roumi como Ministra de Estado da Felicidade, a principal tarefa da ministra foi a de coordenar várias iniciativas de planeamento que mais tarde seriam traduzidas em estratégias e políticas de felicidade. Em 7 de março de 2016, o Sheikh Mohammed bin Rashid Al Maktoum, Vice-Presidente dos Emirados Árabes Unidos e Governante Máximo do Dubai aprovou o Programa Nacional para Felicidade e Bemestar (anteriormente denominado, Programa Nacional para Felicidade e Positividade) apresentado pela Ministra Ohood bint Khalfan Al Roumi (KHAISHGI, 2016; UAEGOV, [s.d.]) .

O Programa Nacional de Felicidade e Bem-estar (PNFBE) traduziu o planeamento da ministra e da sua equipa e estabeleceu as principais políticas de 
felicidade e bem-estar. Os principais objetivos do PNFBE são: 1. inclusão da felicidade nas políticas, programas e serviços de todos os órgãos governamentais e no trabalho; 2. promoção do bem-estar e felicidade como estilo de vida na comunidade; 3 . desenvolvimento de benchmarks e ferramentas para medir a felicidade (UAEGOV, [s.d.]).

Em 20 de março de 2016, o Sheik Mohammed aprovou o Programa Nacional para a Felicidade e definiu-o como a Carta Nacional para a Felicidade. No mesmo dia, o Governante Máximo do Dubai aprovou outras ações para serem implementadas nos organismos governamentais com o objetivo de criar um ambiente de trabalho feliz (KHAISHGI, 2016).

Após uma reorganização governamental, em 2017, a Ministra de Estado da Felicidade tornou-se Ministra de Estado da Felicidade e Bem-estar (UAEGOV, [s.d.]).

Ao estudar a evolução das estratégias de governo e da comunicação governamental, identificamos planos pioneiros e bem estruturados (em constante atualização) que levam ao desenho e implementação de projetos incríveis como a Expo 2020, o 'Museu do Futuro', a 'Missão dos Emirados Marte', a 'Área 2071', a 'Estratégia de Inteligência Artificial dos Emirados Árabes Unidos 2031' ou o 'Plano do Centenário dos Emirados Árabes Unidos 2071' (AL MAKTOUM, 2017). O 'Plano do Centenário 2071' tem a felicidade como um dos quatro pilares:

"uma sociedade feliz e coesa - o desenvolvimento da comunidade é parte integrante do 'Plano do Centenário dos Emirados Árabes Unidos 2071'; os objetivos incluem o estabelecimento de uma sociedade segura, tolerante, coesa e ética que abraça a felicidade e um estilo de vida positivo e uma alta qualidade de vida. O pilar também se concentra no desenvolvimento de programas para preparar as gerações futuras para servir como embaixadores da boa vontade dos Emirados Árabes Unidos, bem como promover a participação das mulheres em todos os setores, tornando os Emirados Árabes Unidos um dos melhores lugares para se viver" (AL MAKTOUM, 2017).

Os cidadãos, com especial enfoque nas gerações futuras, estão no centro das estratégias e de uma grande parte da comunicação do governo. Como vimos noutras iniciativas, essa visão traduz-se em ações específicas e, em alguns casos em documentos políticos. É o caso da publicação da 'Carta dos 50 anos' pelo Sheik Muhammed Al Maktoum ou dos '8 Princípios do Dubai', que na nossa opinião, pode ser considerado um documento-legado, nomeadamente para as próximas gerações. O objetivo do Governante Máximo é inspirar os seus cidadãos a serem felizes e continuar sonhando e trabalhando para prosperidade do país (AL MAKTHOUM, 2018; RIBEIRO, 2020).

Em relação ao ano de 2019, recolheram-se 57 notícias de diferentes fontes, incluindo do website do governo dos Emirados Árabes Unidos. A Tabela 1 apresenta os títulos das notícias que foram recolhidas: 
Tabela 1 - Títulos das notícias do ano de 2019

\begin{tabular}{|c|c|c|c|}
\hline$\#$ & Date & Title & Source \\
\hline 1 & $27 / 01 / 2019$ & Dubai achieves most s us tainable development goals & Gulf News \\
\hline 2 & $10 / 02 / 2019$ & Governments need to be pi voted on happiness, wellbeing: Minis ter & Khaleej Times \\
\hline 3 & $13 / 02 / 2019$ & UAE one of the happies $t$ countries in the world, study reveals & Time Out Dubai \\
\hline 4 & $20 / 02 / 2019$ & A s mart path to urban happiness & Capacity Media \\
\hline 5 & $06 / 03 / 2019$ & \begin{tabular}{|l|l|l|l}
250 & New Schools Join the 'Well Schools Network' to Enhance Positive \\
Education and Wellbeing Principles & & & \\
\end{tabular} & UAE GOV \\
\hline 6 & $06 / 03 / 2019$ & UAE's minis ter of happiness ins is ts her job is no laughing matter & Latimes.Com \\
\hline 7 & 13/03/2019 & UAE launches s tudent s upport groups as part of 'Let's Talk' initiative & Gulfnews \\
\hline 8 & $16 / 03 / 2019$ & $\begin{array}{l}\text { H.H. Sheikh Saif Bin Zayed Al Nahyan Launches'Child Digital Safety' to } \\
\text { enhance children safety and quality of digital life }\end{array}$ & UAE GOV \\
\hline 9 & $16 / 03 / 2019$ & $\begin{array}{l}\text { Sheikh Saif bin Zayed launches 'Child Digital Safety' to enhance children } \\
\text { safety, quality of digital life }\end{array}$ & WAM \\
\hline 10 & 20/03/2019 & UAE expats happiest in region; nation rises in happiness index & Khaleej Times \\
\hline 11 & 20/03/2019 & UAE celebrates International Day of Happiness & Gulf News \\
\hline 12 & 20/03/2019 & International Happiness Day: How Abu Dhabi Airports celebrates & Etur Bon Ews \\
\hline 13 & 24/04/2019 & UAE launches 'Minis try of Pos sibilities' & Business Times \\
\hline 14 & 29/04/2019 & Mohammed bin Rashid honours winners of 'Happiness and Pos itivity Medal' & UAE GOV \\
\hline 15 & $30 / 04 / 2019$ & \begin{tabular}{|l} 
National Program for Happiness and Wellbeing launches the Community \\
Design for Wellbeing Initiative
\end{tabular} & UAE GOV \\
\hline 16 & $05 / 05 / 2019$ & Can AI bring you happiness? Dubai thinks so & Economic Times \\
\hline 17 & 20/05/2019 & Happiness bus for workers launched & Gulf News \\
\hline 18 & $01 / 06 / 2019$ & $\begin{array}{l}\text { Dubai's s mart city initiative is harnessing digital not only to streamline services } \\
\text { and drive economic development but also to spread happiness across the biggest } \\
\text { city in the UAE }\end{array}$ & ACCA Global \\
\hline 19 & $12 / 06 / 2016$ & \begin{tabular}{|l} 
National Wellbeing Strategy 2031 Introduces a New Approach for Government \\
Work
\end{tabular} & EAE GOV \\
\hline 20 & $25 / 06 / 2016$ & The Happiness factor in UAE Real Es tate & Gulf News \\
\hline 21 & $29 / 06 / 2019$ & World Government Summit-Expo 2020 to be the biggest yet & Gulf News \\
\hline 22 & $31 / 07 / 2019$ & Dubai sets its sights on becoming the happiest place on earth & Euronews \\
\hline 23 & 17/08/2019 & 500 Workers Participate in the "Happy S ummer" Initiative in Abu Dhabi & MOREGOV \\
\hline 24 & $26 / 08 / 2019$ & UAE and S audi Arabia to boost cus tomer s ervice at government departments & The National \\
\hline 25 & 28/08/2019 & $\begin{array}{l}\text { National Programme for Happiness concludes interactive camps for promoting } \\
\text { children's digital wellbeing }\end{array}$ & UAE GOV \\
\hline 26 & $11 / 09 / 2019$ & \begin{tabular}{|l|} 
To promote healthy lifes tyle and wellbeing in the UAE National Program for \\
Happiness and Wellbeing launches Nutrition Labelling Policy
\end{tabular} & UAE GOV \\
\hline 27 & $14 / 09 / 2019$ & Revealed: Bonus for $s$ taff of top 5 service centres in the UAE & Gulf News \\
\hline 28 & $02 / 10 / 2019$ & UAE launches Global Hub on Governance for SDGs & \begin{tabular}{|l|l|l}
$\mathbf{W A M}$ \\
\end{tabular} \\
\hline 29 & $25 / 10 / 2019$ & How Finland and the UAE are purs uing happines s & EME Outlook \\
\hline 30 & $09 / 11 / 2019$ & $\begin{array}{l}\text { UAE minis try launches new initiatives for staff, up to one-day off for top } \\
\text { performers }\end{array}$ & Khaleej Times \\
\hline 31 & $18 / 11 / 2019$ & $\begin{array}{l}\text { Al Tayer chairs fourth meeting of Ministry of Possibilities' Department of } \\
\text { Government Procurement }\end{array}$ & WAM \\
\hline 32 & $20 / 11 / 2019$ & $\begin{array}{l}\text { Ohoud Al Roumi participates in Virtual Department of Proactive Services } \\
\text { workshop }\end{array}$ & WAM \\
\hline 33 & S/dATA & Abu Dhabi police launches Traffic Happiness Patrol & $\begin{array}{|lr|}\text { Dmi.A } & \text { E/D } \\
\text { Ubaione/Detail }\end{array}$ \\
\hline 34 & $26 / 11 / 2019$ & Smart Dubai celebrates four years of making Dubai happy & Smart Dubai \\
\hline 35 & $02 / 12 / 2019$ & UAE National Day: Many voices, one pass sion, one nation & Gulf News \\
\hline 36 & $03 / 12 / 2019$ & Mohamed bin Zayed, Sheikhs attend 'March of the Union' & WAM \\
\hline 37 & $06 / 12 / 2019$ & $\begin{array}{l}\text { Looking for a place where a Minis try of Happiness exis ts? Spend a year in Abu } \\
\text { Dhabi }\end{array}$ & Flux Magazine \\
\hline 38 & $11 / 12 / 2019$ & Dubai Ruler leads UAE's most popular Twitter convers ations & WAM \\
\hline 39 & $14 / 12 / 2019$ & Mohammed bin Ras hid, Mohamed bin Zayed announce 2020: Towards the next 50 & WAM \\
\hline 40 & $14 / 12 / 2019$ & UAE improves 10 places in Global Food Security Index & WAM \\
\hline 41 & $15 / 12 / 2019$ & How to get home healthcare services in the UAE & Gulf News \\
\hline 42 & $16 / 12 / 2019$ & Is lamic Conference of Health Minis ters inaugurated & WAM \\
\hline 43 & $16 / 12 / 2019$ & $\begin{array}{l}\text { Family Protection Policy enhances family, community stability initiatives: Hes sa } \\
\text { Essa Buhumaid }\end{array}$ & WAM \\
\hline 44 & $16 / 12 / 2019$ & $\begin{array}{l}\text { Mohamed bin Zayed receives participants of "Journey of the Union } 2019 \\
\text { Initiative" }\end{array}$ & $\mathbf{W A M}$ \\
\hline 45 & $17 / 12 / 2019$ & Res idents can now use more services through UAE Pass & Gulf News \\
\hline 46 & $17 / 12 / 2019$ & $\begin{array}{l}\text { OIC health minis ters conference concludes approving five health-focus ed } \\
\text { decisions }\end{array}$ & $\mathbf{W A M}$ \\
\hline 47 & $18 / 12 / 2019$ & Emirati diplomat says promoting tolerance takes time & AP News \\
\hline 48 & $21 / 12 / 2019$ & UAE: 48 wed at s pecial 'tolerance mas s wedding' & Gulf Nems \\
\hline 49 & 23/12/2019 & UAE Revokes Citizens hip of Dis sidents' Families, Says HRW & Naharnet \\
\hline 50 & 24/12/2019 & No toll outs ide rush hour: All you need to know about Abu Dhabi's road toll gates & Gulf News \\
\hline 51 & $24 / 12 / 2019$ & Minis try of Economy announces plans for SME s ector for 2020 & WAM \\
\hline 52 & 26/12/2019 & Cons umer rights in UAE: This is how the law protects you & Gulfnews \\
\hline 53 & $27 / 12 / 2019$ & Call for action after report reveals high bullying rates in UAE schools & The National \\
\hline 54 & $28 / 12 / 2019$ & DEWA's Childcare Centres win Founding Leader Award & Util Iti Es-Me \\
\hline 55 & $30 / 12 / 2019$ & Special stamps mark 110 years of UAE's postal services & Gulf News \\
\hline 56 & $31 / 12 / 2019$ & If you don't do these classes, you will not get your Labour Card in the UAE & Gulf News \\
\hline 57 & $31 / 12 / 2019$ & The Veil behind Oman's Inves tment Fund & Modern Diplomacy \\
\hline
\end{tabular}


As palavras felicidade, positividade, bem-estar são temas que constam da maioria dos títulos. Embora alguns dos títulos não contenham essas palavras, uma leitura cuidadosa do conteúdo das notícias direciona o leitor para conceitos similares ou relacionáveis.

Como observamos nas notícias dos anos anteriores, em 2019, alguns novos conceitos e iniciativas foram introduzidos sob a liderança do Governo do Dubai e da Ministra da Felicidade. Além disso, é importante destacar a quantidade de iniciativas sobre a felicidade. Em linha com a visão pioneira que já mencionamos, é interessante realçar a criação do Ministério das Possibilidades.

Outra nota importante relaciona-se com o fato de muitos programas e eventos terem sido criados e implementados através da colaboração entre o Ministério da Felicidade e outros Ministérios como o da Saúde ou da Educação, bem como entre outras organizações públicas e privadas. Do ponto de vista do nosso estudo, é importante salientar a cadência de publicação de notícias, revelando que existe uma estratégia de comunicação bem planeada. Outro ponto chave é a inovação constante em termos de programas de médio e longo-prazo, como a 'Estratégia Nacional para o Bem-estar 2031' (ou 'Estratégia 2031) ou (National Strategy for Wellbeing 2031 NSFW 2031), sintetizada na Figura 1:

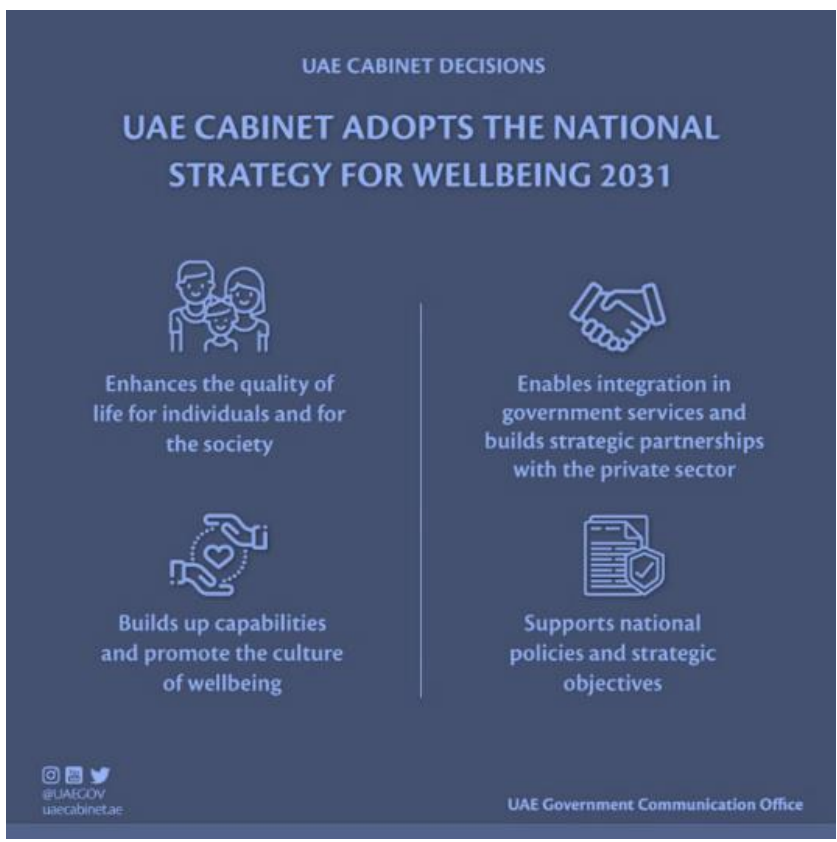

Fig. 1 - Estratégia Nacional para o Bem-estar

Fonte: National Strategy for Wellbeing 2031 - The Official Portal of the UAE Government

De acordo com o Gabinete de Comunicação do Governo dos Emirados Árabes Unidos, o objetivo da Estratégia 2031 é que os Emirados Árabes Unidos se tornem um líder mundial na qualidade de vida. Para ter sucesso, o governo definiu diversos objetivos estratégicos e iniciativas que revelam o seu compromisso sério com o bemestar da sociedade. A Estratégia 2031, pretende tornar-se uma estratégia integrativa que apoia outros programas, como o 'UAE Vision 2031' e o 'UAE Centennial 2071', enquanto promove o bem-estar. A estratégia tem um enquadramento nacional e compreende três níveis principais:

- indivíduos, sociedade e país;

- melhorar o bem-estar das pessoas, promovendo estilos de vida saudáveis e ativos, 
- promover uma boa saúde mental e adotando pensamento positivo.

A Estratégia 2031, compreende ainda 14 componentes e 9 objetivos estratégicos. O conteúdo do documento destaca o objetivo de fortalecer os laços familiares e trabalhar pela melhoria da qualidade de vida em todo o país. Em suma, a Estratégia Nacional para o Bem-estar 2031 é um plano detalhado que abrange quase uma centena de iniciativas de apoio distribuídas por 40 áreas prioritárias. Destas iniciativas constam a criação do primeiro Observatório Nacional do Bem-estar, o lançamento da Academia do Bem-estar para as gerações futuras, e ainda um Conselho Nacional do Bem-estar ao qual é atribuída a tarefa de liderar a implementação desta estratégia inovadora (CABINET, 2019).

\section{Estratégia Digital e a Iniciativa de Segurança Digital Infantil}

O ‘Global Wellness Institute' (Instituto Global do Bem-estar) lançou a 'Iniciativa de Bem-Estar Digital' com o objetivo de reunir líderes influentes de diferentes áreas de conhecimento, nomeadamente: bem-estar, saúde, tecnologia, governo e educação. O objetivo principal da iniciativa é fazer um brainstorm para desenvolver pesquisas e introduzir políticas globais destinadas a educar os cidadãos sobre a era digital. Muitos investigadores e especialistas em todo o mundo já estão a desenvolver estudos sobre as novas tendências e também sobre as problemáticas mais importantes. Algumas das disciplinas centrais do conceito de Bem-Estar Digital são: Ética Digital, Cidadania Digital, Mindfulness Digital, Nutrição Digital e Educação Digital. O 'mundo' do Bem-Estar Digital está nos seus estádios iniciais, revelando um interesse crescente e uma necessidade urgente de abordar o assunto e, acima de tudo, de estabelecer uma visão comum que inter-relacione todas as subdisciplinas. É fundamental que uma estrutura global seja estabelecida com o objetivo de educar e proteger o ser humano no mundo digital, ou seja, "uma abordagem holística e sustentável orientada para o uso e adoção de tecnologia de maneira a que nos permita manter elementos da nossa humanidade não digital na era da tecnologia", conforme referem os responsáveis pelos programas (LUCKOW\&HUBER, [s.d.]).

Na visão do Sheik Al Maktoum: "o ser humano, suas ideias, inovações, sonhos e conexões, são a capital do futuro. Porque para onde vão grandes mentes hoje, grandes coisas acontecerão amanhã" (AL MAKTHOUM, 2018). Nas últimas décadas, os Emirados Árabes Unidos criaram um ambiente único para as pessoas trabalharem juntas por um futuro melhor, com o apoio da tecnologia.

O projeto Smart Dubai tem sido o berço tecnológico de muitos programas inovadores. O planeamento cuidadoso da Smart Dubai foi criado com o objetivo de transformar a cidade para que ela se tornasse uma das melhores Smart Cities com uma tónica evidente na ligação entre tecnologia e felicidade. O planeamento incluiu orientações bem definidas e ações específicas que cuja implementação permitiria extrair lições de melhoria, levando assim à realização do objetivo atrás mencionado (AL-AZZIWI, 2018).

Do nosso ponto de vista, entende-se que será com esta mesma abordagem inovadora e metódica que os Emirados Árabes Unidos trabalharão para a implementação da Iniciativa de Segurança Digital Infantil (ISDI or Child Digital Safety Initiative, CDSI).

De acordo com a Emirates News Agency (WAM), o projeto da Iniciativa de Segurança Digital Infantil foi desenvolvido pelo Ministério do Interior e pelo Programa Nacional de Felicidade (Cf. títulos №. 8 e 9 na Tabela 1). O objetivo da Iniciativa de Segurança Digital Infantil é alertar as crianças sobre os perigos do mundo digital. $O$ projeto alarga-se às famílias pretendendo dar-lhes o suporte necessário para que 
possam e estejam prontas para ajudar os seus filhos. A ISDI foi divulgada no Dia da Criança dos Emirados, em 15 de março, e integra-se num plano mais amplo que visa promover gerações de cidadãos digitalmente alfabetizados (CABINET, 2019).

A iniciativa assume uma grande relevância, especialmente quando se analisam os dados de consumo de tecnologia na região. A mesma fonte (WAM) destaca que os Emirados Árabes Unidos são "líderes mundiais de consumo digital". Os indicadores demonstram o seguinte: $98 \%$ de penetração de internautas em todos os dispositivos; $82 \%$ de penetração de smartphones; $83 \%$ de utilizadores regulares do Whatsapp; 8 horas como tempo médio diário despendido na internet e uma média de 3 horas diárias nas redes sociais (cf. figura 2):

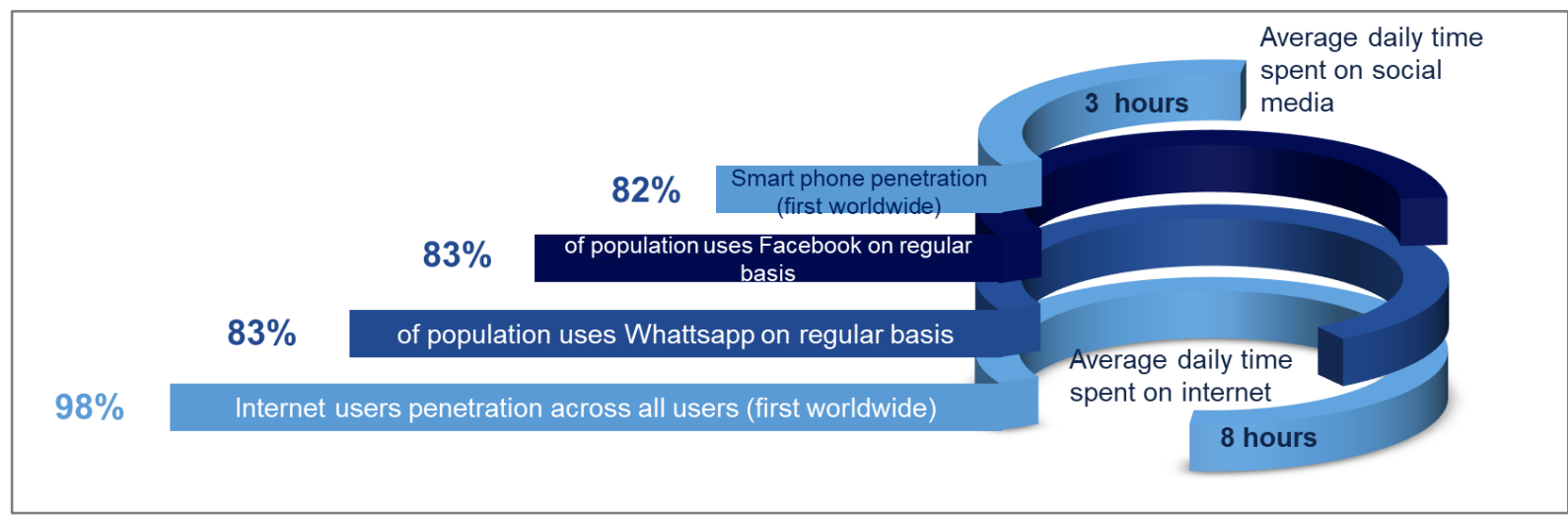

Fig. 2 - Child Digital Safety

Source: Adapted from Gulf News (2019)

Quanto ao conteúdo da ISDI, ele está dividido em 4 sub-iniciativas:

1. Acampamentos infantis interativos;

2. Portal de Bem-Estar Digital;

3. Oficinas de formação;

4. Uma plataforma de apoio para responder a dúvidas urgentes dos pais sobre segurança digital.

A mesma agência de notícias e o Gabinete de Comunição do governo relatam que o Sheikh Saif Bin Zayed Al Nahyan, Vice-Primeiro Ministro e Ministro do Interior dos UAE (discursando durante a apresentação da Iniciativa) disse a própósito deste plano:

"Os Emirados Árabes Unidos são líderes mundiais em penetração de internet e smartphones e isso exige o desenvolvimento de uma rede de segurança digital sofisticada para os nossos filhos. O governo dos Emirados Árabes Unidos está empenhado em manter a estabilidade familiar e a coesão social, e as crianças estão no topo da sua agenda. A sua segurança é fundamental para promover o bem-estar geral nos Emirados Árabes Unidos, mantendo a estabilidade social, capacitando cidadãos positivos e ativos e criando gerações com um domínio firme do mundo digital "(CABINET, 2019).

E, a Sra. Ohood Al Roumi, Ministra de Estado da Felicidade e Bem-estar e Diretora-Geral do Gabinete do Primeiro-Ministro, no mesmo contexto declarou que:

"Melhorar o bem-estar digital é essencial para promover o bem-estar geral na comunidade em geral, e a segurança online é um componente-chave nesse sentido. Para garantir o acesso seguro à internet e a aplicações inteligentes, ao mesmo tempo que se evita o abuso online, os indivíduos 
precisam estar cientes dessas ameaças e estar bem preparados para as enfrentar"(CABINET, 2019).

Em síntese, os textos permitem-nos inferir que o governo dos Emirados Árabes Unidos é capaz não apenas de desenvolver e comunicar políticas conceitualmente abstratas, como a felicidade, mas também de continuar a melhorar e inovar os seus próprios planos e programas, para atingir metas predefinidas em relação a essas políticas. Acima de tudo, o governo dos Emirados Árabes Unidos planeia com antecipação e cria ações específicas para responder aos desafios do mundo digital, neste caso, respeitantes à segurança infantil no mundo digital.

\section{Considerações Finais}

O Governo da Federação dos Emirados Árabes Unidos tem conseguido criar estratégias e políticas inovadoras a partir de conceitos abstratos como felicidade, positividade e, mais recentemente, 'possibilidades' - o Ministério das Possibilidades. Há algumas décadas que o Governo investe nas tecnologias mais avançadas. $O$ projeto Smart Dubai é um exemplo dos projetos cuidadosamente elaborados que o governo criou com sucesso. O mesmo projeto também demonstra como um soft goal (objetivo suave, numa tradução literal) de criar a cidade mais feliz do planeta pode ser trabalhado de uma maneira mais sistematizada, semelhante a uma abordagem da engenharia (AL-AZZIWI, 2018).

Este estudo permitiu identificar vários projetos e programas pioneiros como a Estratégia Nacional de Bem-estar 2031, que inclui a Iniciativa de Segurança Digital Infantil. Os Emirados Árabes Unidos detêm uma posição de liderança em termos de consumo digital, portanto, preparar as gerações futuras e os seus pais para os desafios do mundo digital é uma questão emergente e importante, que no nosso entender deveria ser seguida no resto do mundo.

A "segurança das crianças é fundamental para promover o bem-estar geral nos Emirados Árabes Unidos, manter a estabilidade social, empoderar cidadãos positivos e ativos e criar gerações com um domínio firme do mundo digital", conforme apontado pelo Sheikh Saif Bin Zayed Al Nahyan. Portanto, o governo através da Child Digital Safety Initiative "visa fornecer às crianças as capacidades necessárias e ajudar os pais a compreender as ameaças do mundo digital, juntamente com os meios para combater essas ameaças, a fim de gerir melhor a vida digital dos seus filhos".

O estudo permitiu-nos ainda inferir que o Governo dos Emirados Árabes Unidos e, em particular a Ministra da Felicidade, trabalham ativamente na construção do caminho para a Cidade Mais Feliz do Mundo, sendo a comunicação governamental um fator estratégico para o sucesso das políticas governamentais, conforme demonstrado pela quantidade de notícias publicadas e respetivo conteúdo.

O estudo permitiu ainda evidenciar o foco do governo na felicidade e no bemestar dos cidadãos. Na perspetiva do governo, o bem-estar é também um elemento integrante da Estratégia de Bem-Estar Digital 2031. Na nossa opinião, esta estratégia estabelece a ligação entre as políticas de base subjetiva e a tecnologia, permitindonos compreender como é possível relacionar conceitos abstratos como felicidade e bem-estar com conceitos tecnológicos como o bem-estar digital. Tudo isto está, então, inter-relacionado numa visão mais ampla, traduzida neste plano estratégico.

Por fim, o estudo permite-nos enfatizar a necessidade de uma resposta global da academia e da sociedade à problemática da Segurança Digital Infantil na Era Digital. A Iniciativa de Segurança Digital Infantil dos Emirados Árabes Unidos assumiu a liderança e já está a preparar as gerações futuras para enfrentar e saber gerir os 
riscos digitais emergentes de uma utilização intensiva, com benefícios para as crianças e para as suas famílias e, em última análise, para a sociedade.

Acreditamos que este trabalho constitui um contributo importante para o estudo longitudinal que vimos a realizar desde 2016 sobre a comunicação governamental dos EAU, em particular sobre o Ministério da Felicidade. Entendemos ainda que se trata de uma contribuição importante para chamar a atenção da academia e da sociedade para a problemática da Segurança Digital Infantil na Era Digital.

\section{REFERÊNCIAS}

ABDELATY, $H$. et al. The Relationship between Happiness and Economic Development in KSA: Study of Jazan Region. Asian Social Science, v. 14, n. 3, 2018. AL-AZZIWI, A. Dubai Happiness Agenda: Engineering the Happiest City on Earth. Dubai: [s.n.].

AL MAKTHOUM, M. The Fifty-Year Charter. Dubai's Ex ed. Dubai: [s.n.].

AL MAKTOUM, M. UAE Centennial 2071. Dubai: [s.n.]. Disponível em: $<$ https://u.ae/en/about-the-uae/strategies-initiatives-and-awards/federal-

governments-strategies-and-plans/uae-centennial-2071>.

BAZELEY, P. Qualitative Data Analysis Practical Strategies. Western Sydney University, Australia: SAGE Publications Ltd, 2013.

BHUTAN CENTRE, H. R. A Compass towards a just and harmonious society 2015 GNH Survey Report. Centre for ed. Thimphu, Bhutan: [s.n.].

CABINET, U. National Strategy for Wellbeing 2031. Disponível em: $<$ https://u.ae/en/about-the-uae/strategies-initiatives-and-awards/federal-

governments-strategies-and-plans/national-strategy-for-wellbeing-2031>.

COSTA, A. P.; RIBEIRO, D. "Analysis of Government Communication on the Establishment of the Dubai and United Arab Emirates Ministry of Happiness". TASK2019 - (Towards Advanced Scientific Knowledge in Business Sciences) - - 3a AFU International Conference. Anais...Dubai: 2019

FREY, B. S.; STUTZER, A. What Can Economists Learn from Happiness Research? Journal of Economic Literature, v. 40, n. 2, p. 402-435, jan. 2014.

HELLIWELL, JOHN F., RICHARD LAYARD, JEFFREY SACHS, AND JANEMMANUEL DE NEVE, E. World Happiness Report. New York: [s.n.]. Disponível em: <https://worldhappiness.report/>.

KHAISHGI, A. E. Happiness is a serious job': UAE's Minister of Happiness embraces new role. The National UAE, 2016.

LOOTAH, A. N. Excellence in Implementation': The UAE Model in Reaching the Global Agenda 2030EDA Reflection. Abu Dhabi: [s.n.]. Disponível em: $<$ https://eda.ac.ae/docs/default-source/Publications/eda-reflection_sdgs-2030agenda_en.pdf?sfvrsn=2>.

LUCKOW\&HUBER. Global Wellness Initiative. Disponível em: <https://globalwellnessinstitute.org/initiatives/digital-wellness-initiative/>.

OISHI \& DIENER. Can and should happiness be a policy goal? Health and WellBeing, v. I, p. 195-203, 2014.

RIBEIRO, D., COSTA, A. P., \& REMONDES, J. Comunicação Governamental - A Magna Carta do Dubai. Investigação Qualitativa em Ciências Sociais: avanços e desafios, v. 4, p. 406-421, 2020.

RIBEIRO, C. \& R. Government communication and the 50-year Dubai Charter. 
Journal of Power, Politics \& Governance, v. 8, n. 1, p. 1-14, 2020.

RIBEIRO, D.; COSTA, A. P.; REMONDES, J. Government Communication - The Dubai and United Arab Emirates Ministry of Happiness. In: COSTA, A. P.; REIS, L. P.; MOREIRA, A. (Eds.). . Computer Supported Qualitative Research: New Trends on Qualitative Research (WCQR 2019) - SPRINGER. Porto - Portugal: Springer International Publishing, 2020. p. 226-238.

RIBEIRO, D.; LARANJEIRA, E. Economia da Felicidade - Felicidade $360^{\circ}$ - Modelo para aplicação dos Indicadores FIB no contexto empresarial. ISMAI $1 .{ }^{\circ}$ Congresso Internacional de Ciências Empresariais. Universidade e Empresas: casamento ou divórcio? Anais...Maia - Portugal: 2018

RIBEIRO, D.; REMONDES, J.; COSTA, A. P. Dubai and United Arab Emirates Ministry of Happiness. Presentation of the Happiness and Positivity Programme - Qualitative Analysis. ICSSXXI - 21st INTERNATIONAL CONFERENCE ON SOCIAL SCIENCES. Anais...Amsterdan: 2020

SCHREIER, M. Qualitative Content AnalysisSAGE Publications Inc., , 2008. (Nota técnica).

STIGLITZ, J. E. Report by the Commission on the Measurement of Economic Performance and Social Progress. [s.I: s.n.]. Disponível em: <www.stiglitz-senfitoussi.fr>.

UAE NATIONAL COMMITTEE ON SDGS 2030. UAE and 2030 Agenda for Sustainable Development - Excellence in Implementation. Dubai: [s.n.].

UAEGOV. UAE Ministry of Happiness. Disponível em: <https://u.ae/en/about-theuae/the-uae-government/government-of-future/happiness>.

UAEGOV. United Arab Emirates: 40 Years of Progress - Retrospective Analysis of Key Indicators. Abu Dhabi: [s.n.].

UAEGOV. The UAE portal for the Sustainable Development Goals. Disponível em: $<$ https://uaesdgs.ae/en>. 